\title{
Regime Switching in a Fishery with Stochastic Stock and Price
}

\author{
Linda Nøstbakken*
}

17th May 2005

\begin{abstract}
We develop a bioeconomic model of a fishery subject to stock uncertainty and price uncertainty. With a linear control model, the optimal harvest policy is a bang-bang approach to the optimal stock level, where one harvests either at minimum or full capacity. It is assumed that changing the harvest rate is subject to a switching cost. In this case we show that there are two switching curves in stock-price space, one for entering and one for leaving the fishery. Numerical methods are used to characterize the optimal switching policy for the fishery.
\end{abstract}

\section{Introduction}

Over the last couple of decades there has been an increase in the application of stochastic bioeconomic models in the literature with prices, biological parameters, etc. fluctuating according to stochastic processes. Whereas the literature contains numerous studies of the management of natural resources under some kind of uncertainty, most of them only analyse how one source of uncertainty influences the bioeconomic model. Few studies consider the effects on optimal management of several sources of uncertainty that simultaneously affect different parts of the bioeconomic model.

The purpose of this study is to analyse how uncertainty in stock growth and price influence the optimal harvest of fish. We build our model on the well-known deterministic linear-control model presented by Clark \& Munro (1975). When the stock reaches the critical level, harvest is set at some interior value, which maintains the optimal stock

*Norwegian School for Economics and Business Administration, N-5045 Bergen, Norway. Email: linda.nostbakken@nhh.no 
level (steady state). Uncertainty is incorporated into the model by letting price and stock-recruitment evolve according to known stochastic processes. The Clark-Munro model is further extended through the introduction of fleet switching costs. It seems reasonable to assume that increasing and decreasing the harvest rate incurs certain costs. We therefore assume lump-sum switching costs of changing the harvest rate. It will be shown that the optimal harvest is either to harvest at minimum or full capacity. While it is not possible to find a closed form solution to the optimisation problem, numerical methods can be used to approximate the solution. These will be used to characterise the optimal policy, which is defined by regime-switching curves in stock-price space; one for activating the fishing fleet and one for withdrawing the fleet from the fishery.

Of related work, Clarke \& Reed (1989) and Reed \& Clarke (1990) introduced price and growth uncertainty in a forest harvest model, modelling the price process as geometric Brownian motion and assuming stock growth to be age or size dependent. Brennan \& Schwartz (1985) present a model where a project, a mine is used as an example, can operate in two modes; active or passive. There is one output, and the output price fluctuates according to a known stochastic process. The payoff from the project depends on the current output price and on the choice of output rate. Switching between the active and the passive modes is done at a fixed cost. Brennan and Schwartz are able to derive expressions for the value of the project. They also consider optimal management of the mine. In his study of entry and exit decisions under uncertainty, Dixit (1989) builds on the analysis in Brennan \& Schwartz (1985). Dixit makes several simplifications to the Brennan and Schwartz model, such as assuming a fixed production rate in the active state. This allows him to derive analytical results. In a recent work, Lumley \& Zervos (2001) analyse optimal investment in a non-renewable natural resource industry subject to switching costs.

The theory on real options has been developed over the past two decades. The real option theory involves treating investment projects as options to invest, while the investment projects can be anything from job search or whether to open a factory, to the exploitation of natural resources. Financial economics offer techniques to price options and to determine optimal exercise time or state. The real options approach is therefore a convenient way to analyse investment projects and is especially valuable when analysing projects involving uncertainty. The literature on real options contains many examples of optimal switching models. Dixit \& Pindyck (1994) give a good introduction to real options and they present several models of optimal switching. Other examples of optimal switching models can be 
found in Trigeorgis (1996), and in the recent collection of Schwartz \& Trigeorgis (2001).

The remainder of the paper is organised as follows. The next section specifies the bioeconomic model. The numerical analysis, where we characterise the optimal policy, is presented in section 3. A summary and conclusions are presented in section 4 .

\section{Model Specification}

Let the fish stock at instant $t$ be denoted by $X=X(t)$, where time is continuous, with $\infty>t \geq 0$. Instantaneous harvest from the stock, the harvest rate, is denoted by $Y=Y(t)$. There is an upper limit to how much the fishing fleet can harvest at every instant of time given by the maximum harvest rate $Y_{\max }$. We therefore have $Y_{\max } \geq Y \geq 0$. We assume the dynamics of the resource stock $X$ is given by:

$$
d X=[F(X)-Y] d t+\sigma_{X} X d z_{X}
$$

where $F(X)$ is a strictly concave growth function, with $F(0)=F(K)=$ 0 and where $K>0$ is the carrying capacity of the environment. The term $\sigma_{X} X d z_{X}$ represents the stochastic part of the stock-growth relationship and can be thought of as random environmental fluctuations. $\sigma_{X} X$ is the standard deviation rate, $\sigma_{X}>0, d z_{X}=\epsilon(t) \sqrt{d t}$, where $\epsilon(t)$ is a standard normal, iid, random variable. It follows that $z(t)$ is a Wiener process. Clark \& Munro (1975) explain growth by an equation similar to the deterministic part of equation (1).

The price of the resource, $P$, is assumed to follow a process of geometric Brownian motion (GBM), given by:

$$
d P=\mu P d t+\sigma_{P} P d z_{P}
$$

where $\mu \geq 0$ is the drift rate and $\sigma_{P} P$ is the standard deviation rate, $\sigma_{P}>0 . \quad d z_{P}=\epsilon(t) \sqrt{d t}$ is also an increment of a Wiener process. We further assume that $E\left\{d z_{X}, d z_{P}\right\}=0$, i.e., we are dealing with a small fishery whose harvest has no affect on the world price, $P$. A positive drift rate implies that prices are increasing over time, perhaps reflecting a growing demand for fish protein or a decline in stocks, world-wide.

We make the standard assumption that an agent seeks to maximise the expected present value of net revenues from the fishery over an infinite horizon subject to the dynamic constraints given by equations (1) and (2). ${ }^{1}$ Assume the cost per unit fish harvested is $c(X)$, with

\footnotetext{
${ }^{1}$ Fixed costs are disregarded as we assume the fleet in question participates in other,
} 
$c^{\prime}(X)<0$ and $c^{\prime \prime}(X)>0$. If there is no uncertainty, i.e., if $\sigma_{X}=\sigma_{P}=$ 0 , and if the price is constant $(\mu=0)$, the model is exactly the same as the Clark-Munro model (1975). The optimal control problem is

$$
J(X, P)=\max _{\{Y\}} E\left\{\int_{s}^{\infty}(P-c(X)) Y e^{-\rho t} d t\right\}
$$

where $\rho$ is the discount rate, subject to (1) and (2). The optimal harvest will therefore, at every instant of time, follow the most rapid approach path (MRAP) toward the optimal stock level. The optimal harvest is consequently either $Y=0$ or $Y=Y_{\max }$. In the deterministic case $\left(\sigma_{X}=\sigma_{P}=0\right)$ with constant price, the optimal harvest rate is first a bang-bang approach to the optimal stock level, then a constant harvest rate to maintain the optimal steady state.

We will assume that a switching cost is incurred for any change in harvest rate. The cost of increasing the harvest rate is $A_{12}$ and the cost of decreasing the harvest rate is $A_{21}$. This changes the original problem and one might question if the MRAP solution is still optimal. Let $\epsilon_{i}$ and $\tau_{j}$, where $i=1, \ldots, n$ and $j=1, \ldots, m$, be the times of increases and decreases in harvest rate, respectively. The net present value of the cost of the $n+m$ changes in harvest rate must now be subtracted from the expression given by equation (3). The control problem is nevertheless still linear in harvest and is therefore maximised by the MRAP solution. Any approach other than bang-bang lowers the expected discounted value of net revenues. This implies that as long as it is optimal to vary the harvest rate at all, it is optimal to switch between zero and $Y_{\max }$ since the cost of doing so is the same as the cost of switching between interior harvest rates $\left(Y_{\max }>Y>0\right)$. Hence, the MRAP solution maximises the expected value of the fishery subject to switching costs if the MRAP solution is also better than harvesting at a constant harvest rate at all times. This is what we investigate next.

Let the fishery be partially open at all times with a fixed harvest rate of $m Y_{\max }(1>m>0)$. By choosing this strategy switching costs are avoided. The corresponding stochastic control problem can be expressed as

$$
J(X, P)=\sup _{m} E\left\{\int_{s}^{\infty}(P-c(X)) m Y_{\max } e^{-\rho t} d t\right\}
$$

where $d X=\left[F(X)-m Y_{\max }\right] d t+\sigma_{X} X d z_{X}$, while $d P$ as before is given by equation (2). The Hamilton-Jacobi-Bellman equation for the

and to the fleet, more important fisheries. 
problem stated in equation (4) is

$$
\begin{aligned}
\rho V=\sup _{m}\{ & (P-c(X)) m Y_{\max }+\left(F(X)-m Y_{\max }\right) V_{X}+\mu P V_{P} \\
+ & \left.\frac{1}{2} \sigma_{X}^{2} X^{2} V_{X X}+\frac{1}{2} \sigma_{P}^{2} P^{2} V_{P P}\right\}
\end{aligned}
$$

The maximum condition for the problem is

$$
\frac{\partial\{\cdot\}}{\partial m}=\left(P-c(X)-V_{X}\right) Y_{\max }=0
$$

and we see that the supremum cannot be reached for any value of $m$. This implies that the optimal solution to the profit maximisation problem is a bang-bang solution. The optimal harvest rate is consequently either $Y=0$ or $Y=Y_{\max }$ and we can define two regimes; $R=1$, inactive, and $R=2$, active (harvesting at the rate $\left.Y=Y_{\max }\right)^{2}$

Brekke \& Øksendal (1994) characterise the solution of a switching model, which encompasses the current one. Using stochastic calculus, they derive conditions for the optimal solution to the problem and prove the existence of a solution. The optimal value function $V(X, P, R)$ for the fisheries management problem must satisfy. ${ }^{3}$

$$
\begin{aligned}
\rho V(X, P, R) & \geq \pi(X, P, R)+[F(X)-Y] V_{X}(X, P, R) \\
& +\mu P V_{P}(X, P, R)+0.5 \sigma_{X}^{2} X^{2} V_{X X}(X, P, R) \\
& +0.5 \sigma_{P}^{2} P^{2} V_{P P}(X, P, R)
\end{aligned}
$$

and the condition

$$
V(X, P, R) \geq V(X, P, i)-A_{R i}, R \neq i
$$

where $R \in(1,2)$ is regime, and $\pi(X, P, R)=(P-c(X)) Y_{\max }(R-1)$ is the flow of net revenues per unit of time from harvesting the stock in regimes 1 and 2. The left-hand side of equation (5) is the fishery's opportunity cost in regime $R$, while the right-hand side, which gives the sum of instantaneous net revenues and value gain from changes in price and stock, is the return rate in regime $R$. The condition given by equation (6) states that the value of remaining in regime $R$ must be at least as high as the value of regime switching, given by the value

\footnotetext{
${ }^{2}$ Since it could become optimal to harvest the stock to extinction the harvest rate is also limited by the available stock, i.e., $Y=\left\{0, \min \left(Y_{\max }, X\right)\right\}$.

${ }^{3}$ Dixit \& Pindyck (1994) provide necessary and sufficient conditions to similar real options problems.
} 
of being in the other regime minus the cost of switching. In addition to the conditions given by equations (5) and (6), the optimal value function must satisfy some regularity conditions, namely the value matching and smooth pasting conditions. These regularity conditions are given by equations (7) and (8), respectively.

$$
\begin{gathered}
V\left(X_{\text {entry }}, P_{\text {entry }}, 1\right)=V\left(X_{\text {entry }}, P_{\text {entry }}, 2\right)+A_{21} \\
V\left(X_{\text {exit }}, P_{\text {exit }}, 2\right)=V\left(X_{\text {exit }}, P_{\text {exit }}, 1\right)+A_{12} \\
V_{X}\left(X_{i}, P_{i}, 1\right)=V_{X}\left(X_{i}, P_{i}, 2\right), \text { for } i=\{\text { entry, exit }\} \\
V_{P}\left(X_{i}, P_{i}, 2\right)=V_{P}\left(X_{i}, P_{i}, 1\right), \text { for } i=\{\text { entry, exit }\}
\end{gathered}
$$

The optimal policy can be defined by switching curves in stockprice space. If $A_{12}>0$ and/or $A_{21}>0$, there are two switching curves in $X-P$ space; one for entering the fishery (moving from regime 1 to regime 2) and one for leaving the fishery (moving from regime 2 to regime 1). The switching curves are implied by equations (5) and (6). In regime 1 , one is indifferent between entering the fishery and staying inactive if $V(X, P, 1)=V(X, P, 2)-A_{12}$. This defines the entry curve. Similarly, in regime 2 one is indifferent between leaving and staying active if $V(X, P, 2)=V(X, P, 1)-A_{21}$, which defines the exit curve. Between the two switching curves both harvesting and inactivity can be optimal depending on what one is currently doing; the optimal behaviour is to remain passive. The higher the switching costs, the larger the area between the switching curves and the less frequent would be switches by the fleet. If $V(X, P, 1)>V(X, P, 2)$, inactivity is always optimal, whereas if $V(X, P, 1)<V(X, P, 2)$ it is always optimal to harvest. Dixit (1989) describes how the presence of uncertainty and switching costs can result in hysteresis, which he defines as "the failure of an effect to reverse itself as its underlying cause is reversed". This inertia explains why there are two switching curves in $X-P$ space and not one as would be the case in a fishery without switching costs. In the next section numerical methods are used to approximate optimal switching curves for the fishery.

\section{Numerical Analysis ${ }^{4}$}

The optimality conditions, along with regularity conditions can be used to numerically approximate the optimal switching curves for our

\footnotetext{
${ }^{4}$ The numerical analysis utilises the CompEcon Toolbox (for Matlab) as developed by Miranda \& Fackler (2002). The Matlab code used in this section can be made available by the author upon request.
} 
problem. In this section we first study the long-run distribution of biomass when there is no harvesting. Next, optimal switching curves for the fishery are approximated and the optimal management fishery is simulated and characterised.

Before we can initiate the numerical analysis we need to make assumptions about the specific forms of the cost and growth functions and we must specify model parameters. First, we assume a cost per unit of harvest of $c(X) \equiv \frac{c}{X}$, a unit cost function which corresponds to the Schaefer production function and a constant cost of $c$ per unit effort. Second, stock growth is assumed to follow the logistic growth function $F(X)=r X\left(1-\frac{X}{K}\right)$, where $r$ is the intrinsic growth rate. Parameter values are summarised in Table 1 . Note that the maximum harvest rate is set to $Y_{\max }=0.25$. This is above the maximum sustainable yield of $M S Y=\frac{r K}{4}=0.125$ and it is thus impossible to harvest at full capacity at all times without driving the stock to extinction. We assume no drift in the price of fish, i.e., $\mu=0$. For most commercial fish stocks this seems to be a reasonable assumption. The assumption does however not affect our results in any significant way. The same is the case for the assumption of $A_{12}=A_{21}$; the equality is not necessary and does not qualitatively change the results.

Table 1: Parameter Values

\begin{tabular}{ccc}
\hline Parameter & Value & Description \\
\hline$r$ & 0.5 & Intrinsic growth rate \\
$K$ & 1 & Biological carrying capacity \\
$c$ & 0.25 & Cost per unit effort \\
$Y_{\max }$ & 0.25 & Upper bound harvest rate \\
$\mu$ & 0 & Price drift \\
$\sigma_{P}$ & 0.2 & Price diffusion \\
$\sigma_{X}$ & 0.3 & Stock diffusion \\
$\delta$ & 0.1 & Discount rate \\
$A_{12}$ & 0.01 & Cost of increasing harvest rate \\
$A_{21}$ & 0.01 & Cost of decreasing harvest rate \\
\hline
\end{tabular}

The long-run distribution of the pristine stock is found numerically using equation (1) with $Y=0$, the logistic growth function, and parameter values as presented in Table 1.

Figure 3 shows the long-run density functions for the base case $\left(\sigma_{X}=0.3\right)$ and for standard deviation rates 0.15 and 0.45 . Dixit \& Pindyck (1994) note that $E\{X\}=K\left(1-\sigma_{X}^{2} /(2 r)\right)$. The stock density varies significantly with the value of the standard deviation 
Figure 1: Long-Run Stock Density, $Y=0$. Base case $\sigma_{X}=0.30$ (solid line), $\sigma_{X}=0.45$ (dash-dot line), and $\sigma_{X}=0.15$ (dashed line).

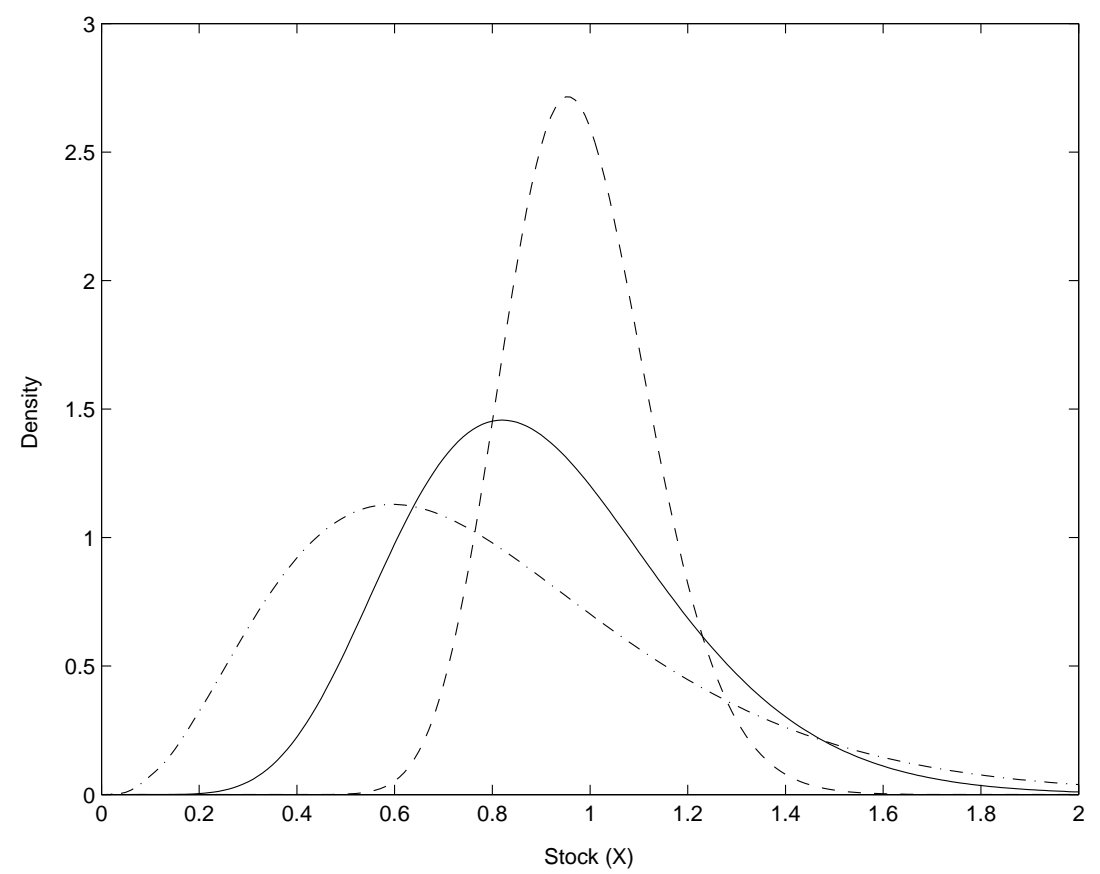

rate $\sigma_{X}$. For $\sigma_{X}=0.3$, the base case, the vast majority of stock realisations are within the interval 0.15 to 2 , where 2 is twice the size of the carrying capacity. The stock can in theory go extinct even in the no-fishing case but the likelihood of this is approximately zero.

\subsection{Optimal Switching Curves}

Optimal switching curves for the fishery are found using a cubic spline approximation function. A spline can be described as any smooth function that is piecewise polynomial but also smooth where the polynomial pieces connect (see e.g. Judd, 1998). A cubic spline is constructed of piecewise third-order polynomials and produces continuous first and second derivatives. A Matlab procedure described in Fackler (2004) is used to obtain the numerical solution. The procedure uses function approximation and collocation to find the optimal solution characterised by the optimality conditions (5) and (6). In addition to these, we know that the fishery is valueless if the stock goes extinct and therefore add the condition $V(0, P, R)=0$, which must hold for all $P$ and $R$.

Approximate Switching Curves. Base case (thin lines) and with 


\section{Figure 2: Approximate Switching Curves. Base case (thin lines) and with No switching costs (thick line).}

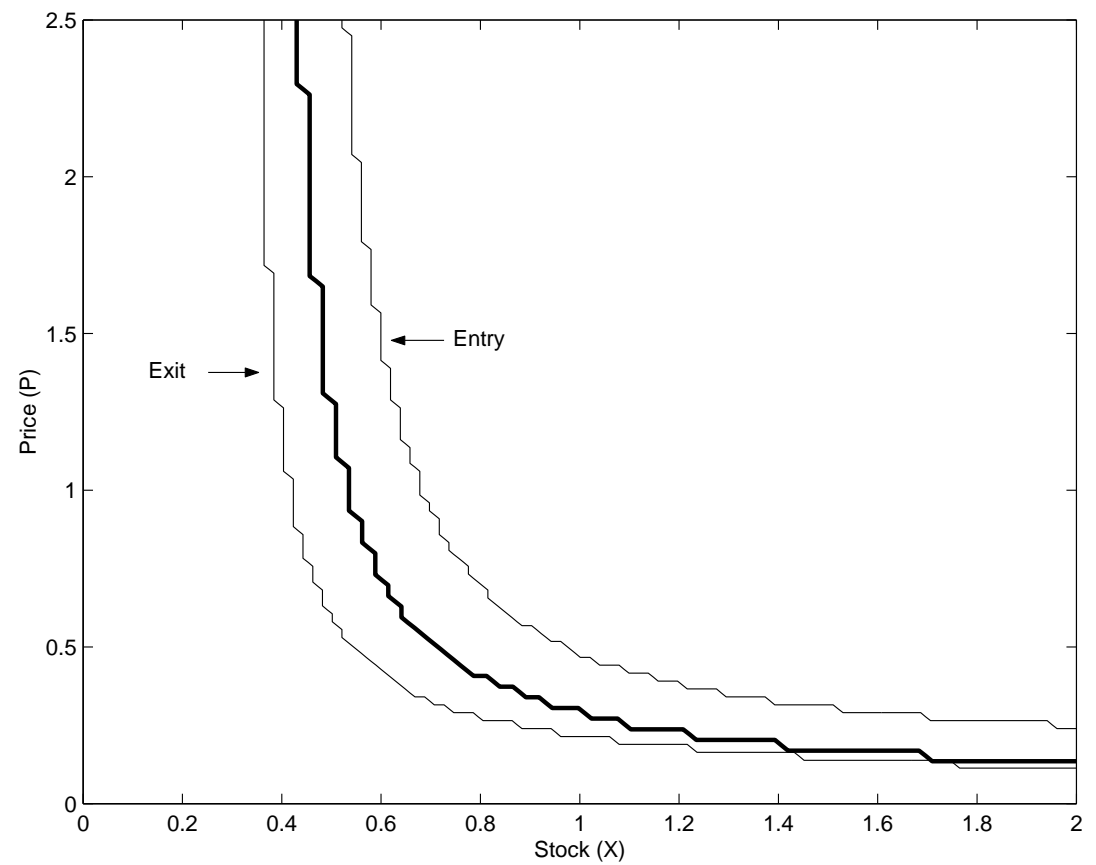

No switching costs (thick line). The approximate optimal switching curves can be seen in Figure 3.1. The two curves labelled entry and exit are the optimal switching curves in the base case (positive switching costs). The third curve in Figure 3.1 represents the case of no switching cost. The continuous state space must be discretised into a finite set of state nodes when approximating the optimal policy and this explains why the curves are not smooth. For $X, 100$ evenly spaced points on the interval $[0,2]$ are used to make the grid of state nodes. Considering the long-run stock distribution (Figure 3), we see that the interval $2 \geq X \geq 0.15$ covers virtually every possible stock realisation. The long-run distribution of price, on the other hand, depends on initial price. For $P, 100$ evenly spaced points on the interval $[0,2.5]$ are chosen when defining the grid of state nodes.

Obviously, as price increases, it gets more and more profitable to harvest the stock, everything else being equal (Figure 3.1). The same is true as stock increases since the unit cost of harvesting is decreasing in stock size. Accordingly, it is always optimal to harvest when price is high and stock is high, while inactivity, or no harvest, is optimal if both variables are at low levels. In other cases the switching curves reflect the fact that there are trade offs between getting a high (low) 
price and having a low (high) stock. The sensitivity of the switching curves to changes in either of the two state variables decreases with the value of the state variable.

The switching curve representing entry into the fishery lies above the exit curve. It follows that there is an area between the two curves where fishing can be optimal or suboptimal, depending on the current regime. This is the band of hysteresis, as described earlier. The distance between the switching curves depends on the switching costs. If there are no switching costs there is only one curve, as can be seen in Figure 3.1. As the switching costs increase, the distance between the curves grows. Eventually, at very high switching costs, there will be no switching curves since no possible realisations of price and stock exist where harvest revenues would make up for the switching costs.

To our best knowledge, this is the first study of optimal switching curves in a fishery with stochastic stock and price.

\subsection{The Optimal Management Fishery}

The long-run consequences of following the optimal switching policy are found by stochastic simulations. The policy given by the base-case switching curves described above are simulated 2,000 times over $T=$ 5,000 time increments, where each time increment is set to $d t=0.001$. Initial values of price and stock are 1 and 0.5 , respectively. Sample realisations of price and stock are shown in Figure 3.2. The stock level is higher when the price is low than when price is high. This, of course, is because the stock is being harvested down whenever it is high enough to justify activating the fleet. The higher the price, the sooner activation becomes optimal. The long-run density of stock is shown in Figure 3.2 along with the density of the pristine stock.

According to the simulation results, a moratorium is in effect in the fishery most of the time. The average harvest at time increment $T$ shows that a positive harvest rate $(Y)$ is optimal only $45 \%$ of the time. The rest of the time the fishery is closed. A different choice of parameter values alters this. Nevertheless, the conclusion that the fishery stays closed a large share of the time does not come as a total surprise. Remember that we set the maximum harvest rate to $Y_{\max }$. This is twice the maximum sustainable yield rate of 0.125 . As a consequence it takes little time to harvest the stock down from above the entry curve to below the exit curve. It can take considerably more time for the stock to grow back up to a level above the entry curve and as a result, the fishery is closed a large part of the time. What we find is referred to as pulse fishing in the literature (see e.g. Hannesson, 1975). Pulse fishing has been shown to be optimal in several cases 


\section{Figure 3: Sample Realisations of Price and Stock.}
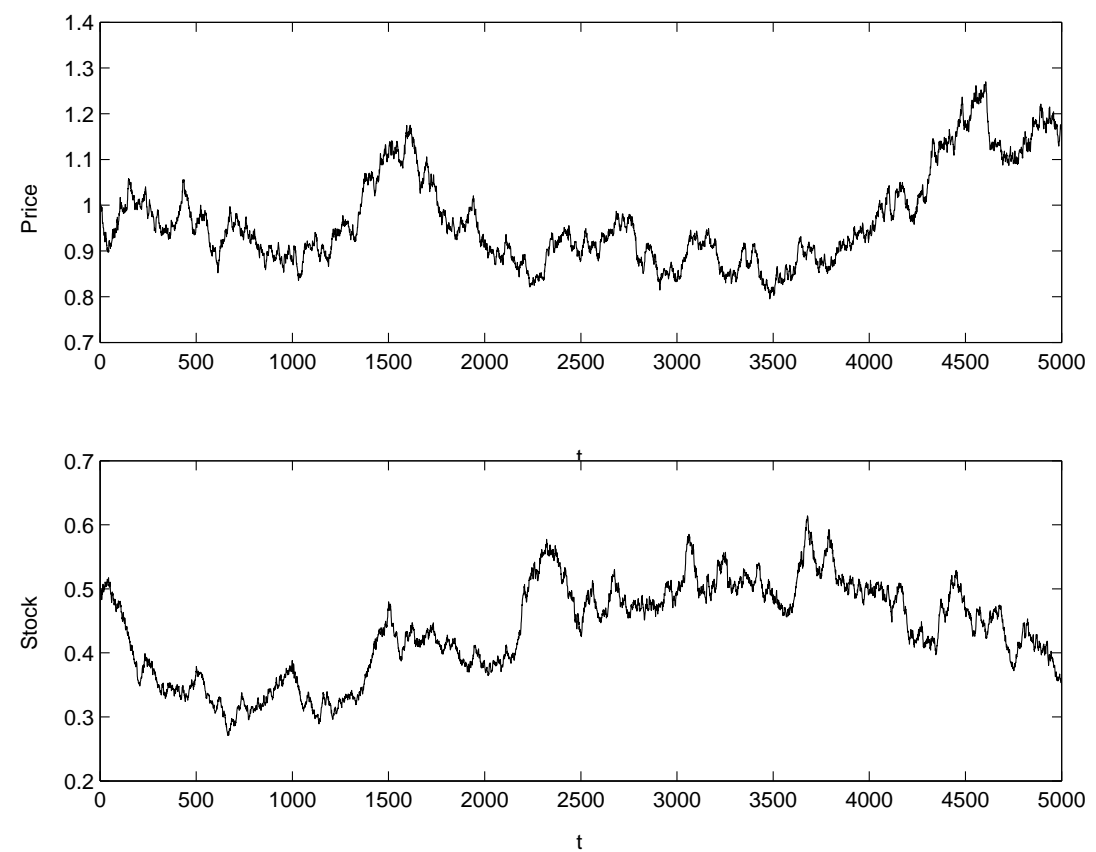

where the control model is linear, i.e., when the cost of increasing capacity in the fishery is linear. If, on the other hand, we were dealing with a non-linear control problem, pulse-fishing would have been more surprising.

We set out to approximate optimal switching curves for the fishery and to study the implications of applying the optimal policy, as defined by the switching curves. With this in place, we can analyse the sensitivity of the solutions to parameter changes. This is what we turn to next.

\subsection{Sensitivity Analysis}

We start out by evaluating the case analysed in Clark \& Munro (1975). Steady-state conditions are easily derived for the deterministic case without fleet-adjustment costs. This case will therefore be used as a benchmark. We also analyse how changes in stock and price volatilities $\left(\sigma_{i}, i=X, P\right)$ affect the switching curves, how sensitive the curves are to changes in growth rate, and finally, how changes in the maximum harvest rate affect the results presented in the previous section.

In the deterministic case with fixed price and no switching costs, there is an optimal steady-state stock level $X^{*}$ realised at a certain 
Figure 4: Long-Run Stock Density: Optimal Policy and No Harvesting (dashed line).

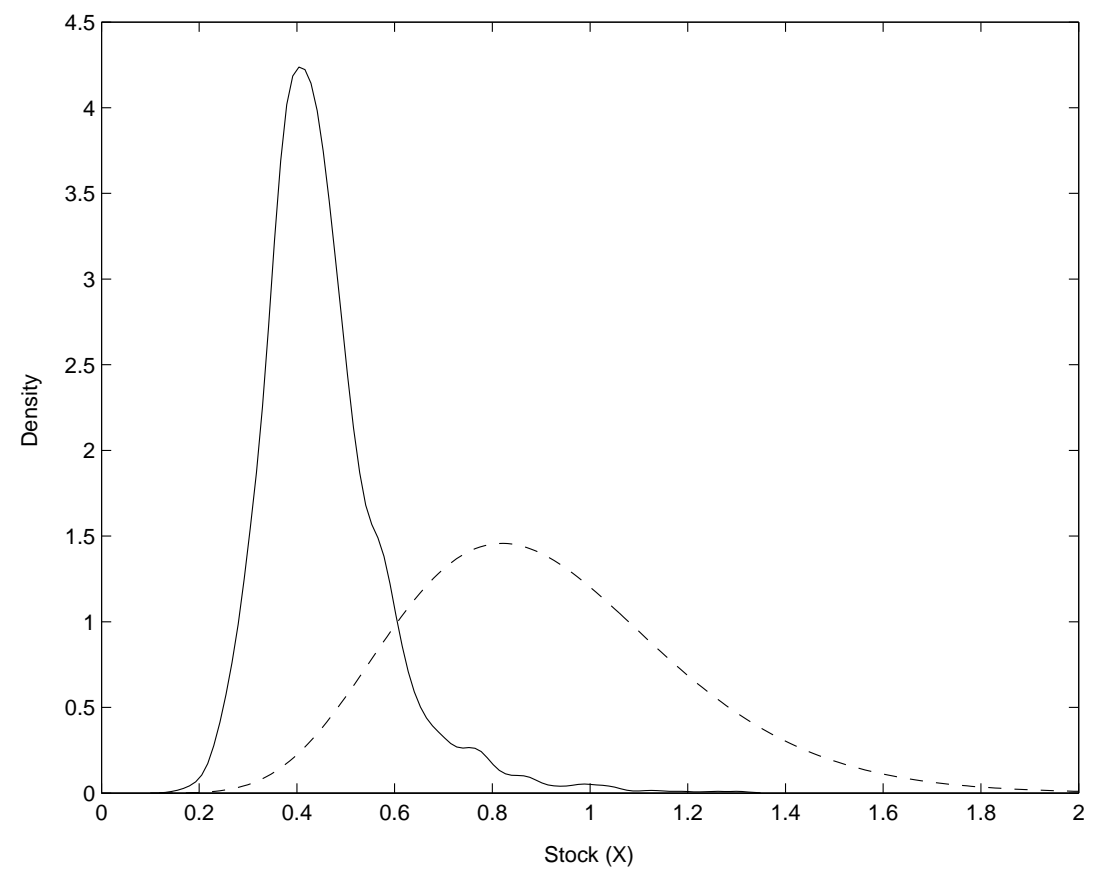

harvest rate $Y^{*} \in\left[0, Y_{\max }\right]$. In steady state, harvest is set at some interior value which maintains the optimal stock level. Steady state stock and harvest rate are given by

$$
\begin{aligned}
P-c\left(X^{*}\right) & =\frac{F\left(X^{*}\right) c^{\prime}\left(X^{*}\right)}{F^{\prime}\left(X^{*}\right)-\rho} \\
Y^{*} & =F\left(X^{*}\right)
\end{aligned}
$$

Based on the first equation we can calculate steady-state combinations of stock and price which can be represented as a curve in stock-price space. This curve is similar to the switching curves shown in Figure 3.1 for the stochastic case. If the fishery is not currently in steady state one should, depending on the current state, harvest either at $Y_{\max }$ or not at all until steady state is reached. In the deterministic case there will only be one switching curve even if fleet adjustments are subject to switching costs. When one knows everything there is no need to make fleet adjustments after the optimal steady state is reached. One will switch at most once and as a result the switching costs do not change anything. 
From the steady-state relationship we know it is not optimal to harvest at any positive, finite price for stock levels $X \leq(r-\delta) \frac{K}{2 r}$. This means that the stock must be above 0.4 in our fishery for there to exist a positive steady-state harvest rate. Further, if price is less than $P=\frac{c}{K}(0.25)$ fishing is not viable at any stock level $X \in[0, K]$. In comparison, we have established that harvesting in a stochastic setting can be optimal at stock levels well below 0.4 and finite prices (see Figure 3.1). Similarly, as stock increases it can be optimal for the fleet to stay active even though price is less than 0.25 and stock is 1 . The reason is inertia; because of the uncertainty, it is better to keep the fleet active for a while in case things take a better turn, than paying the switching cost. The sensitivity of the switching curves with respect to price and stock volatility is presented in Figure 3.3. The degree of volatility does not affect the switching curves much. Note also how the deterministic steady-state curve matches the exit curve at high stock levels, and the entry curve at high price levels.

Figure 5: Approximate Switching Curves. Base Case (thick lines), Deterministic (thick dash-dot line), High Volatility with $\sigma_{X}=0.45$ and $\sigma_{P}=0.30$ (thin dashed lines), and Low Volatility with $\sigma_{X}=0.15$ and $\sigma_{P}=0.10$ (thin lines).

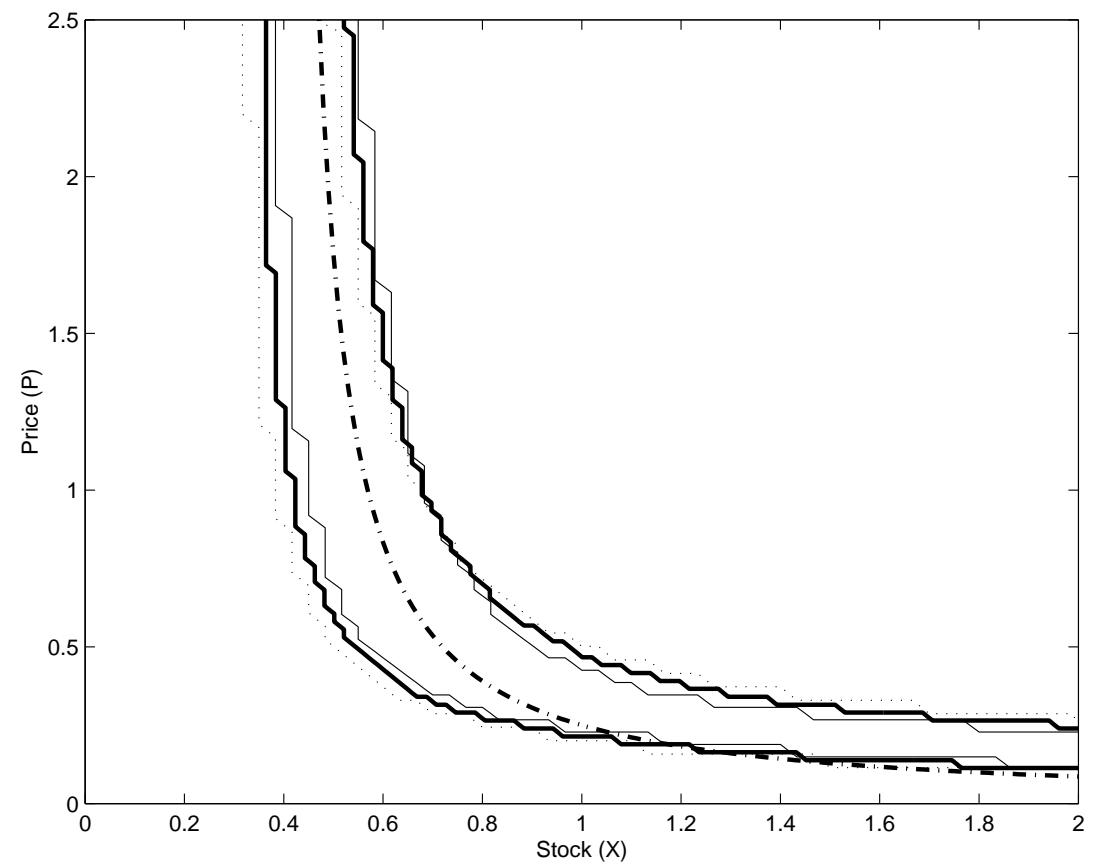

The growth rate of the stock limits the fishing fleet's harvest. The higher the intrinsic growth rate, the higher the maximum sustainable 
Figure 6: Approximate Switching Curves. Base case $r=0.5$ (thin lines), $r=0.25$ (thick line), and $r=0.75$ (thick dash-dot lines).

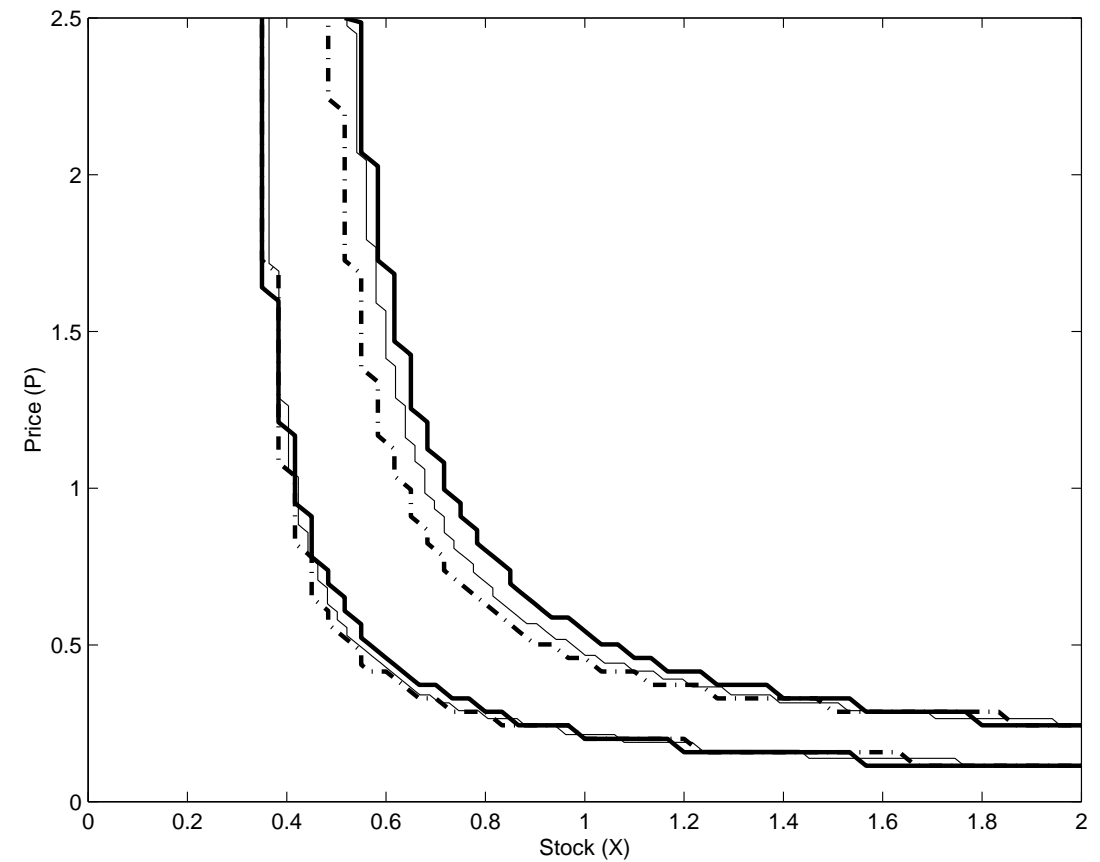

yield. Figure 3.3 shows approximate switching curves for alternative growth rates. The exit curve is fairly insensitive to changes in growth rate. The entry curve, on the other hand, changes with the growth rate - the higher the growth rate, the closer the entry curve is to the exit curve. This means that the fleet is active a larger share of the time when the growth rate is high, other things being equal. This is reasonable since a higher growth rate means a larger stock growth and more fish available in the sea for harvesting. At high stock levels, the growth rate affects the switching curves very little.

So far we have assumed that we are dealing with a fishing fleet capable of harvesting at a rate twice as high as the maximum sustainable yield rate. In many fisheries, however, the capacity of the fishing fleet is not nearly as high as this and it is therefore interesting to see what happens to the fishery as we reduce the maximum harvest rate of the fleet. New switching curves are approximated for the fishery with maximum harvest rates 0.15 and 0.05 , the latter being well below the maximum sustainable harvest rate. Other parameters are as presented in Table 1. As can be seen in Figure 3.3, a reduction in $Y_{\max }$ has little effect on the switching curves for high stock levels $(X>0.9)$. For lower levels of the stock, however, the change is significant and 
Figure 7: Approximate Switching Curves. Base case (thin lines), $Y_{\max }=0.15$ (thick line), and $Y_{\max }=0.05$ (thick dash-dot lines).

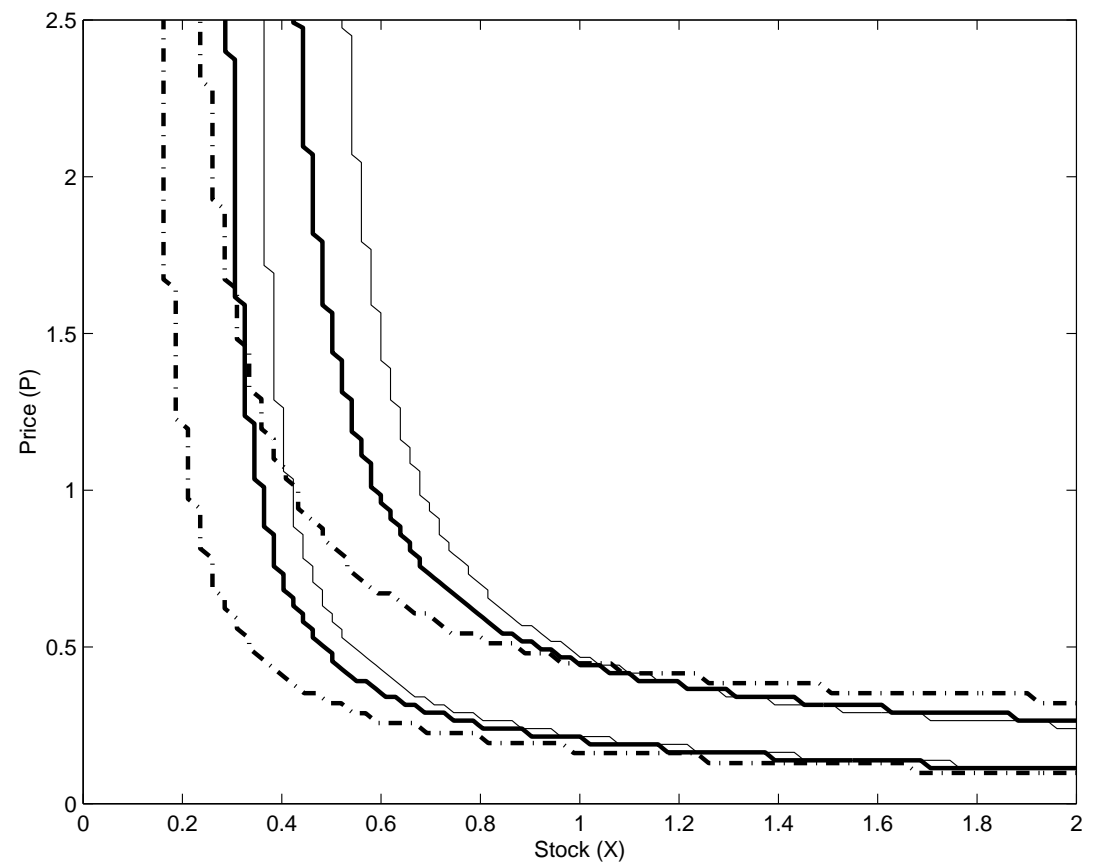

the smaller the maximum harvest rate, the closer the switching curves are to the price axis. The difference in sensitivity between high and low stock levels can be explained by the fact that the value of having a large stock is limited when the maximum harvest rate is small. This is similar to what Hannesson (1993) finds in his example of the capelin fishery. In addition, we see that the distance between the entry and exit curves seems to increase with $Y_{\max }$. In a deterministic setting a maximum harvest rate of 0.05 would result in a constant harvest at full capacity for price $P \geq 0.60$. Simulations of the stochastic fishery (with $X_{0}=0.5$ and $P_{0}=1$ ) show that something similar happens there; the fleet will be active approximately $98 \%$ of the time when $Y_{\max }=0.05$. This corresponds to an average stock of 0.71 .

Table 2 summarises the sensitivity statistics. The variation in stock level is seen to decrease with the maximum rate of harvest. The difference in long-run average biomass between the cases $Y_{\max }=0.15$ and $Y_{\max }=0.25$ is relatively small, which makes the fairly large difference in variation noteworthy. A larger maximum harvest rate enables the fleet to faster bring the stock back to its desired level when something changes. We are however dealing with a fleet that harvests either at $Y_{\max }$ or not at all, and a large $Y_{\max }$ could therefore cause increased 
Table 2: Fishery Characteristics by Maximum Harvest Rate, $Y_{\max }$

\begin{tabular}{cccc}
\hline & $Y_{\max }=0.05$ & $Y_{\max }=0.15$ & $Y_{\max }=0.25$ \\
\hline Fishery open, share of time & $98 \%$ & $69 \%$ & $45 \%$ \\
Biomass, mean & 0.71 & 0.49 & 0.45 \\
Biomass, standard deviation & 0.28 & 0.19 & 0.12 \\
\hline
\end{tabular}

volatility in biomass.

\section{Summary and Conclusions}

In this study we have seen how entry and exit curves can be computed when both the stock and the price of a natural resource evolve stochastically and when there are costs to changing the harvest rate. The production function, which was used to explain harvesting in the model, is linear in effort. Under the assumption of a constant cost per unit effort, we end up with a linear control problem. When maximising the total present value of net revenues over an infinite horizon, the optimal policy is consequently to harvest at full or at minimum capacity. The optimal policy has been defined by switching curves in stock-price space. By making the additional assumption that changing the harvest rate is subject to switching costs, we show that there exist two curves in stock-price space, one for activating the fleet and one for withdrawing the fleet from the fishery. These curves are numerically approximated and we study the fishery when employing the optimal switching policy. In a deterministic setting, the switch from harvesting at full or minimum capacity to maintaining an optimal stock level (steady state) occurs at most once with price constant. With stock and price uncertainty there is no steady state and it is optimal to switch back and forth between minimum and maximum harvest rates. The dual switching curves are a result of the combination of uncertainty and switching costs in the model.

Based on our initial choice of parameter values, we find that pulse fishing is the optimal behaviour of the fishing fleet and that it is optimal for the fishery to stay closed most of the time. Looking at the sensitivity of our results to parameter changes, we see that price and stock volatilities do not affect the switching curves much. The maximum harvest rate of the fishing fleet, on the other hand, significantly affects the optimal switching curves. Further, it turns out that one of the effects of having a larger harvesting capacity is a more stable 
stock, even under "bang-bang" harvest policy.

Many fisheries are being harvested by fleets, which also take part in other fisheries. From time to time, when the conditions allow for it, the fleet enters a particular fishery, harvests the stock down, before, once again, moving on to other fisheries. Such fisheries serve to illustrate the relevance of the regime-switching model defined and analysed in this paper. While we study a single fishery and assume lump-sum costs to increasing and decreasing the harvest rate, the resulting optimal behaviour, pulse fishing, is found in many real-world fisheries.

The current analysis can be extended in several ways. First, we assume a fixed capacity, which is reflected in the constant maximum harvest rate of the fishing fleet. The analysis can be extended by incorporating capacity as a third state variable (c.f. the deterministic model by Clark et al., 1979). When capital is subject to depreciation, the manager must decide upon an optimal investment policy in addition to the optimal harvest policy. This extension would however increase the complexity of the analysis dramatically. Second, the cost of changing the harvest rate can be assumed to increase with the magnitude of the adjustment made in fishing fleet or harvest rate. This will perhaps give a more accurate depiction of the reality in most fisheries. Another possibility for future research is to apply the model to a real-world fishery. 


\section{References}

Brekke, K. A. \& B. Øksendal (1994) Optimal switching in an economic activity under uncertainty. SIAM Journal on Control and Optimization 32, 1021-1036.

Brennan, M. \& E. Schwartz (1985) Evaluating natural resource investments. Journal of Business 58, 135-157.

Clark, C. W., F. H. Clarke, \& G. R. Munro (1979) The optimal exploitation of renewable resource stocks: Problems of irreversible investment. Econometrica 47, 25-47.

Clark, C. W. \& G. R. Munro (1975) The economics of fishing and modern capital theory: A simplified approach. Journal of Environmental Economics and Management 2, 92-106.

Clarke, H. R. \& W. J. Reed (1989) The three-cutting problem in a stochastic environment: The case of age-dependent growth. Journal of Economic Dynamics and Control 13, 569-595.

Dixit, A. K. (1989) Entry and exit decisions under uncertainty. The Journal of Political Economy 97, 620-638.

Dixit, A. K. \& R. S. Pindyck (1994) Investment under uncertaintyPrinceton, New Jersey: Princeton University Press.

Fackler, P. L. (2004) Solving optimal switching models. Downloaded from http://www4.ncsu.edu/ pfackler/.

Hannesson, R. (1975) Fishery dynamics: A north-atlantic cod fishery. The Canadian Journal of Economics 8, 151-173.

Hannesson, R. (1993) Bioeconomic analysis of fisheriesOxford: Fishing News Book.

Judd, K. L. (1998) Numerical Methods in EconomicsCambridge, Massachusetts: MIT Press.

Lumley, R. \& M. Zervos (2001) A model for investments in the natural resource industry with switching costs. Mathematics of Operations Research 26, 637-653.

Miranda, M. J. \& P. L. Fackler (2002) Applied computational economics and financeCambridge, Massachusetts: MIT Press. 
Reed, W. J. \& H. R. Clarke (1990) Harvest decisions and asset valuation for biological resources exhibiting size-dependent stochastic growth. International Economic Review 31, 147-169.

Schwartz, E. \& L. Trigeorgis (2001) Real Options and Investment under Uncertainty: Classical Readings and Recent ContributionsCambridge, Massachusetts: MIT Press.

Trigeorgis, L. (1996) Real Options: Managerial Flexibility and Strategy in Resource AllocationCambridge, Massachusetts: MIT Press. 